\title{
Study of Cerium-modified Triazinedithiol Electrodeposited Nanofilm on Corrosion Protection for Aluminum Alloy
}

\author{
Wang $F^{*}$, Wang J, Jia M, Shi M and Zhang F \\ College of Science, Northwest A\&F University, Xinong Road No.22, Yangling, Shaanxi, 712100, China
}

\begin{abstract}
The cerium-modified polymeric nanofilm of 6-(N,N-dibutyl)amino-1,3,5-triazine-2,4-dithiol monosodium (DBN) was fabricated by two-step potential electrodeposition onto aluminum alloy for corrosion protection. The structure, surface wettability and corrosion protection of the polymeric nanofilm were investigated by means of fourier transform infrared spectroscopy (FT-IR), water contact angle (WCA), open-circuit potential (OCP), potentiodynamic polarization and electrochemical impedance spectroscopy (EIS), respectively. FT-IR result revealed that DBN monomer had successfully been polymerized on aluminum alloy surface by electrochemical deposition in the absence and presence of cerium and the addition of cerium had no influence on FT-IR of the polymeric nanofilm. The result of surface wettability showed that WCA of aluminum alloy cerium-modified polymeric nanofilm was slightly higher compared with that without cerium. Electrochemical measurements demonstrated that the cerium-modified polymeric nanofilm on aluminum alloy surface exhibited remarkable corrosion protection property, which could be ascribed to the precipitation of cerium oxides or hydroxides on aluminum surface or the coordination between cerium and heterocyclic $\pi-e l e c t r o n$ in polymeric nanofilm to prevent corrosive particles to the surface of aluminum substrate.
\end{abstract}

Keywords: Aluminum alloy; Cerium-modified; Electrodeposition; Polymeric nanofilm; Corrosion protection

\section{Introduction}

Due to good adhesion, anti-corrosion, superhydrophobicity, lubrication and dielectric property on a variety of metal substrates, the application of triazinedithiol compounds has attracted many researchers' attention during the past decade [1-11]. Especially, as the anti-corrosion materials, the triazinedithiol polymeric nanofilm exhibits basic corrosion resistance [12]. Unfortunately, the polymeric nanofilms fabricated by traditional techniques $[4,7-9,13]$ have the propensity for containing molecule-sized defects to some extent, which limits its application in more fields. In order to reduce the defects in the polymeric nanofilm, some other methods have been extensively studied in the past few years, such as the change of the substituent on triazinedithiol's structure [12], the combination of self-assembled method and electrodeposition [10] as well as the preparation of complex nanofilm [11]. These techniques enhanced the anti-corrosion property for metals to some degree.

It is well known that cerium ion as corrosion inhibitor doped in nanofilm can inhibit the corrosion of metal substrates and improve anti-corrosion property because of its self-healing ability [14-17]. Although the mechanism is not fully clear, some publications are still reported using cerium such as $\mathrm{Ce}\left(\mathrm{NO}_{3}\right)_{3}$ to enhance the corrosion resistance of the nanofilms $[18,19]$. However, the techniques mentioned above to dope foreign cerium components mostly focused on utilizing silane agents as matrix to prepare functional films. As a promising environmentally coating, it has not been reported so far to dope cerium into the triazinedithiol polymeric nanofilm. It is known that triazinedithiol compounds have triazine ring which presents electronegativity, while cerium shows electropositivity. It could be assumed that triazine ring and cerium might combine with each other by a weak electrostatic force or coordinative bond during the electrodeposited process to obtain the denser hybrid nanofilm. When the metal corrosion occurred, cerium might come out to product the precipitation of cerium hydroxides or cerium oxides to protect the substrate in the vicinity of the cathodic sites, where $\mathrm{OH}^{-}$ions were formed from the oxygen reduction reaction. Therefore, incorporating cerium into triazinedithiol nanofilm is believed to enhance anticorrosion performance.

In this paper, we chose AA5052 aluminum alloy as substrate and 6-(N,N-dibutyl)amino-1,3,5-triazine-2,4-dithiol monosodium (DBN) as triazinedithiol monomer to fabricate the polymeric nanofilm of DBN with the aid of electrochemical technique. Meanwhile, $\mathrm{Ce}\left(\mathrm{NO}_{3}\right)_{3}$ as inhibitor was adopted to prepare cerium-doped polymeric nanofilm by two-step potential electrodeposition. The structure and surface wettability of those polymeric nanofilms were studied by fourier transform infrared spectroscopy (FT-IR) and water contact angle measurements (WCA). The corrosion resistance of those nanofilms on aluminum alloy were investigated by open-circuit potential $(\mathrm{OCP})$, potentiodynamic polarization and electrochemical impedance spectroscopy (EIS).

\section{Experimental}

\section{Materials and reagents}

The substrate for this study was AA5052 aluminum alloy with the dimension of $30 \mathrm{~mm} \times 50 \mathrm{~mm} \times 0.3 \mathrm{~mm}$ and the chemical compositions of AA5052 were shown in Table 1 . All test plates were ultrasonically

\begin{tabular}{|l|l|l|l|l|l|l|l|}
\hline $\mathrm{Cu}$ & $\mathrm{Si}$ & $\mathrm{Fe}$ & $\mathrm{Mn}$ & $\mathrm{Mg}$ & $\mathrm{Zn}$ & $\mathrm{Cr}$ & other \\
\hline $0.1 \%$ & $0.2 \%$ & $0.4 \%$ & $0.1 \%$ & $2.8 \%$ & $0.1 \%$ & $0.3 \%$ & $0.15 \%$ \\
\hline
\end{tabular}

Table 1: Chemical composition for aluminum alloy AA5052 (mass fraction).

*Corresponding author: Wang F, College of Science, Northwest A\&F University, Xinong Road No.22, Yangling, Shaanxi, 712100, China, Tel: +86-29-87092226; Fax: +86-29-87092226; E-mail: wangfang4070@nwsuaf.edu.cn

Received January 15, 2015; Accepted March 12, 2015; Published March 24 2015

Citation: Wang F, Wang J, Jia M, Shi M, Zhang F (2015) Study of Cerium-modified Triazinedithiol Electrodeposited Nanofilm on Corrosion Protection for Aluminum Alloy. J Material Sci Eng 4: 159. doi:10.4172/2169-0022.1000159

Copyright: ( 2015 Wang F, et al. This is an open-access article distributed under the terms of the Creative Commons Attribution License, which permits unrestricted use, distribution, and reproduction in any medium, provided the original author and source are credited. 
degreased with acetone for $15 \mathrm{~min}$ and blow-dried with warm air, followed by corona treatment $(50 \mathrm{~V}, 2.0 \mathrm{~A})$ for $20 \mathrm{~s}$. DBN monomer was synthesized by the reaction of 1,3,5-triazine-2,4,6-trichloride with dibutyl amine and $\mathrm{NaSH}$, according to the method described in the previous paper [20]. The molecular structure of DBN was shown in Figure 1. All of the chemicals were employed as analytical reagents without further purification. Distilled water was used as solvent and $\mathrm{NaNO}_{2}$ was applied as supporting electrolyte for electrodeposition. Cerium nitrate $\left(\mathrm{Ce}\left(\mathrm{NO}_{3}\right)_{3}\right.$, purity $\left.\geq 99.0 \%\right)$ was used as adding component to modify electrodeposited nanofilm. The concentrations of DBN, $\mathrm{NaNO}_{2}$ and $\mathrm{Ce}\left(\mathrm{NO}_{3}\right)_{3}$ were kept constant at $5 \mathrm{mM}, 0.15 \mathrm{M}$ and $0.5 \mathrm{mM}$, respectively.

\section{Preparation of electrodeposited polymeric nanofilm}

The electrodeposition was performed by using a three-electrode cell, with the AA5052 alloy plate as a working electrode, the saturated calomel electrode (SCE) as a reference electrode and the stainless steel plate as counter electrode. The electrodeposited polymeric nanofilm of DBN was prepared by two-step potentiostat method using electrochemical workstation (CHI 660C, CH Instrument, Shanghai, China) on AA5052 surface. The first step potential was $1.6 \mathrm{~V}$ and $6.0 \mathrm{~V}$ was applied for the second step. Electrodeposition time corresponded to two-step potential was $30 \mathrm{~s}$ and $10 \mathrm{~s}$, respectively. After the electropolymerization, the samples were taken out from the electrolytic cell and immediately rinsed by distilled water and acetone, then blowdried with warm air. Finally, the samples were cured at $100^{\circ} \mathrm{C}$ for 10 min in an oven.

\section{Characterization of electrodeposited nanofilm}

FT-IR was carried out by attenuated total reflection spectroscopy (Bruker TENSOR 37) in the range of 4,000 to $600 \mathrm{~cm}^{-1}$. WCA was measured by optical contact angle measuring instrument (SL100) at room temperature with $2 \mu \mathrm{L}$ distilled water. OCP was performed for $1200 \mathrm{~s}$ by electrochemical workstation in $0.5 \mathrm{M}$ sodium chloride $(\mathrm{NaCl})$ aqueous solution. Potentiodynamic polarization were conducted from $-0.9 \mathrm{~V}$ to $-0.1 \mathrm{~V}$ with the scanning rate of $0.5 \mathrm{mV} / \mathrm{s}$ in $0.5 \mathrm{M} \mathrm{NaCl}$ aqueous solution for pitting corrosion study or in $1 \mathrm{M} \mathrm{H}_{2} \mathrm{SO}_{4}$ solution for uniform corrosion study, respectively. EIS was recorded over a frequency range from $10 \mathrm{mHz}$ to $100 \mathrm{kHz}$ with the excitation voltage of $10 \mathrm{mV}$ after 1 hour immersion time in $0.1 \mathrm{M} \mathrm{NaCl}$ solution under open-circuit potential.

\section{Results and Discussion}

\section{FT-IR spectra analysis}

FT-IR spectra measurements were conducted by reflection absorption and potassium bromide ( $\mathrm{KBr}$ ) method to investigate the change of chemical structure from monomer (a) to polymeric nanofilm

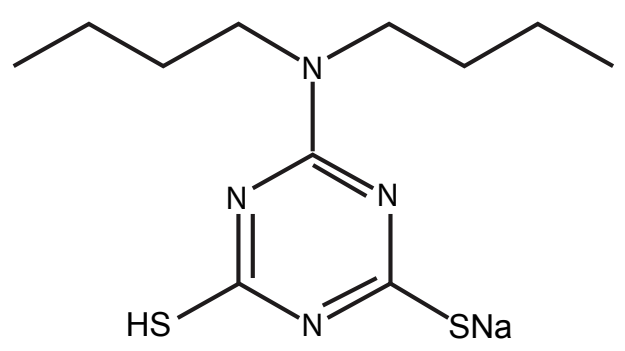

Figure 1: The molecular structures of DBN.

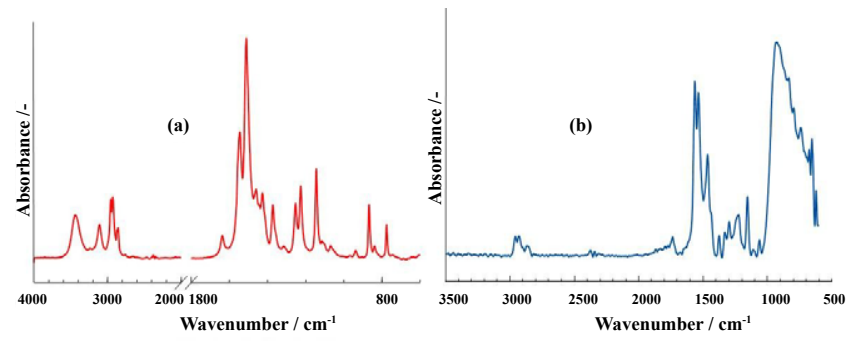

Figure 2: FT-IR spectra of monomer (a, by means of $\mathrm{KBr}$ method) and polymeric film (b) of DBN on aluminum alloy surface.

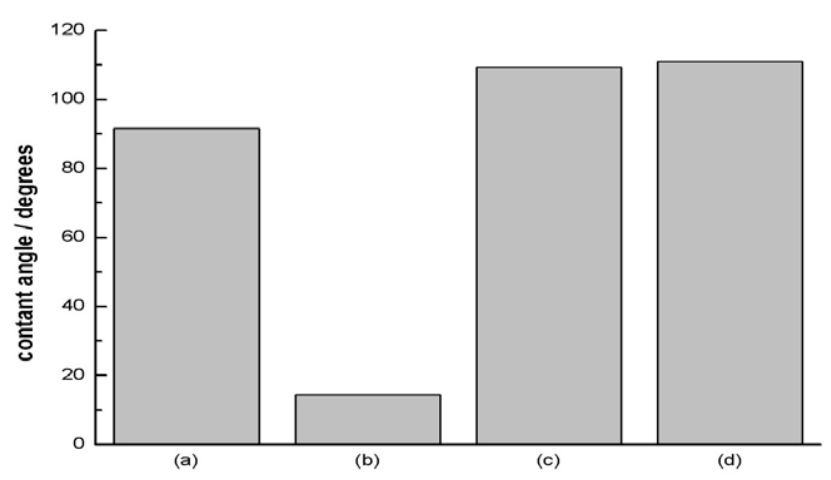

Figure 3: WCA of AA5052 surface. (a) blank; (b) corona discharge treated; (c) polymeric nanofilm in the absence of cerium (III); (d) polymeric nanofilm modified by cerium (III).

(b) of DBN as showed in Figure 2. In Figure 2a, the presence of triazine ring was confirmed by the absorption peaks at $1564 \mathrm{~cm}^{-1}, 1536 \mathrm{~cm}^{-1}$ and $1465 \mathrm{~cm}^{-1}>\mathrm{C}=\mathrm{N}$ - bonds and the absorption peaks at $1375 \mathrm{~cm}^{-1}$ and $1331 \mathrm{~cm}^{-1}$ due to C-N bonds. Dibutyl amino groups were confirmed by the absorption peaks at $2959 \mathrm{~cm}^{-1}$ and $2931 \mathrm{~cm}^{-1}$ due to C-H asymmetric stretching vibrations of $-\mathrm{CH}_{2}$ and $-\mathrm{CH}_{3}$ and the absorption peak at 2868 $\mathrm{cm}^{-1}$ due to $\mathrm{C}-\mathrm{H}$ symmetric stretching vibrations of $-\mathrm{CH}_{2}$. Meanwhile, the peaks at $3410 \mathrm{~cm}^{-1}$ and $3161 \mathrm{~cm}^{-1}$ were clearly detected, which were assigned to the stretching vibration of $\mathrm{N}-\mathrm{H}$. After electrochemical polymerization (Figure $2 \mathrm{~b}$ ), the absence of peak at $3410 \mathrm{~cm}^{-1}$ and 3161 $\mathrm{cm}^{-1}$ and the presence of a new broaden strong absorption peak at 926 $\mathrm{cm}^{-1}$ were observed, which could ascribe to the formation of S-Al or $\mathrm{O}-\mathrm{Al}$ on AA5052 surface. However, for the cerium modified polymeric nanofilm, FT-IR spectroscopy did not give any obvious difference in the structure (data not shown), suggesting that the addition of cerium did not influence the production of polymeric nanofilm. The above results revealed that polymeric nanofilms were formed on AA5052 substrate surface by electropolymerization of DBN no matter whether cerium was added or not.

\section{Contact angle measurement}

WCA were usually used to characterize the wettability of material surface. To confirm the formation of polymeric nanofilms in the absence and presence of cerium, WCA of aluminum surface was measured before and after the formation of nanofilms as shown in Figure 3. WCA of the blank aluminum alloy surface was $91.5^{\circ}$, while WCA of the blank substrate after corona discharge treatment sharply dropped to $14.4^{\circ}$, suggesting that the pretreated blank aluminum alloy had been converted into a hydrophilic substrate from a weak hydrophobic substrate. It can be attributed to the destruction of natural oxidation film on aluminum 
alloy surface and the formation of hydrophilic group after corona discharge treatment. For the electrodeposited nanofilm of DBN in the absence of cerium, WCA increased to $109.3^{\circ}$ and was up to $111.4^{\circ}$ for the electrodeposited nanofilm in the presence of cerium. These results demonstrated that DBN monomers were electropolymerized on aluminum substrate surface to form the polymeric nanofilms, which resulted in the improvement of the hydrophobicity of the surface. Furthermore, the electrodeposited polymeric nanofilm modified by cerium (III) had slightly higher hydrophobicity. Humid environment is one reason of aluminum alloy corrosion, and the hydrophobic surface of substrate is benefit for the protection of aluminum alloy [11]. It was proposed that electrodeposited polymeric nanofilms in the absence or presence of cerium could have excellent protective effect for substrate surface.

\section{Open-circuit potential (OCP)}

Figure 4 displayed a comparison of the OCP of blank, electrodeposited polymeric nanofilm covered the substrate surfaces in the absence and presence of cerium (III) in $0.5 \mathrm{M} \mathrm{NaCl}$ solution for $1200 \mathrm{~s}$. For the corrosion system, the OCP and the corrosion potential were the basic problem for the corrosion phenomena. In general, the more negative the OCP, the better the cathodic protection effect. The potential measurement clearly demonstrated a negative shift in OCP for AA5052 covered by electrodeposited polymeric nanofilms in the absence and presence of cerium (III). This behavior could be attributed to protective effect of the electrodeposited polymeric nanofilms on the aluminum surface that slowed down the cathodic reaction of substrate. Furthermore, OCP of the electrode covered by cerium-doped polymeric nanofilm was more negative than that without cerium-doped nanofilm, clearly suggesting the cathodic protection effect of cerium component.

\section{Potentiodynamic polarization}

To study the resistance to pitting corrosion of these electrodeposited nanofilms for aluminum alloy surface, the potentiodynamic polarization test was performed in $0.5 \mathrm{M} \mathrm{NaCl}$ aqueous solution as shown in Figure 5. The electrochemical parameters such as corrosion potential $\left(\mathrm{E}_{\text {corr }}\right)$, corrosion current density $\left(\mathrm{I}_{\text {corr }}\right)$ and anodic/cathodic tafel slopes $(\beta$ and $\beta_{c}$ ) which were determined by the tafel extrapolation method, were presented in Table 2. According to the approximate linear

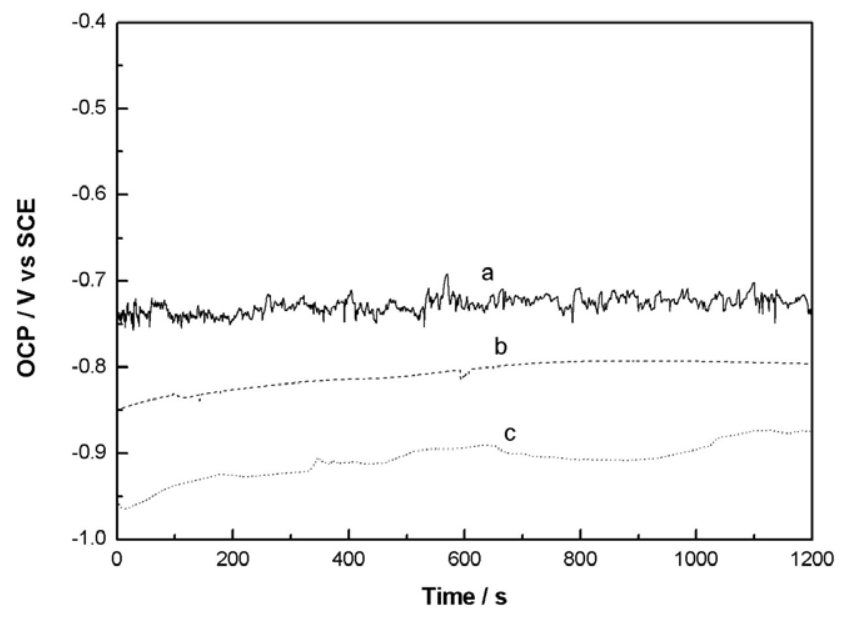

Figure 4: Comparison of the OCP of blank (a), electrodeposited polymeric nanofilm covered the substrate surface without cerium (III) (b) and electrodeposited polymeric nanofilm covered the substrate surface with cerium (III) in $0.5 \mathrm{MNaCl}$ solution (c).

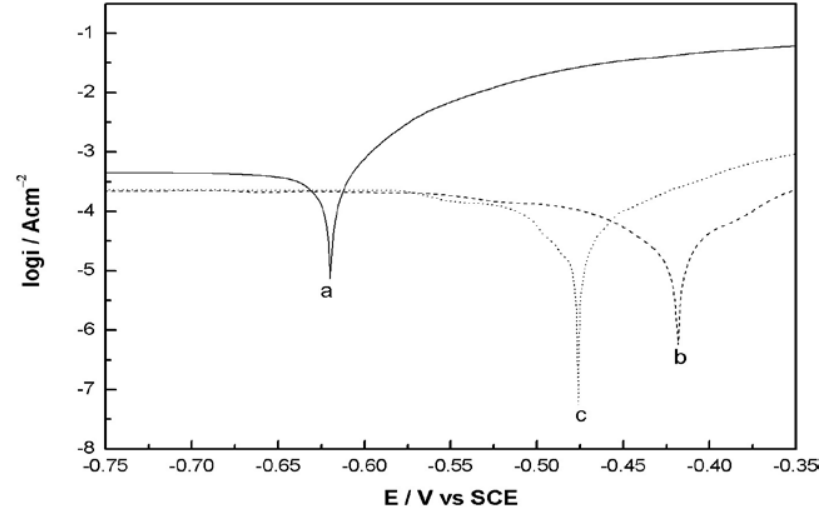

Figure 5: Polarization curves after $20 \mathrm{~min}$ exposure in $0.5 \mathrm{MNaCl}$. (a) blank; (b) electrodeposited polymeric nanofilm covered AA5052 without cerium (III) (c) electrodeposited polymeric nanofilm covered AA5052 with cerium (III).

\begin{tabular}{|l|l|l|l|l|l|l|}
\hline Sample & $\mathbf{E}_{\text {corr }}(\mathbf{V})$ & $\mathbf{I}_{\text {corr }}\left(\mathbf{A} / \mathbf{c m}^{2}\right)$ & $\boldsymbol{\beta}_{\mathrm{a}}$ & $\boldsymbol{\beta}_{\mathrm{c}}$ & $\mathbf{R}_{\mathrm{p}}\left(\mathbf{\Omega} / \mathbf{c m}^{2}\right)$ & $\mathbf{P E}$ \\
\hline (a) & -0.620 & $1.21 \times 10^{-3}$ & 9.27 & 0.315 & 37.6 & - \\
\hline (b) & -0.418 & $8.73 \times 10^{-5}$ & 7.898 & 2.537 & 477.1 & $92.7 \%$ \\
\hline (c) & -0.485 & $5.54 \times 10^{-5}$ & 9.458 & 3.898 & 587.7 & $95.5 \%$ \\
\hline
\end{tabular}

Table 2: Electrochemical parameters after 20 min exposure in $0.5 \mathrm{M} \mathrm{NaCl}$. (a) blank; (b) electrodeposited polymeric nanofilm covered AA5052 without cerium (III); (c) electrodeposited polymeric nanofilm covered AA5052 with cerium (III).

polarization, the corrosion potential $\left(\mathrm{E}_{\text {corr }}\right)$, polarization resistance $\left(\mathrm{R}_{\mathrm{p}}\right)$ and protection efficiency (PE) could be obtained by the following expressions [21,22].

$$
\begin{aligned}
& \mathrm{R}_{P}=\beta_{\mathrm{a}} \beta_{\mathrm{c}} / 2.3 \mathrm{I}_{\text {corr }}\left(\beta_{\mathrm{a}}+\beta_{\mathrm{c}}\right) \\
& \mathrm{PE}=\left(1-\mathrm{I}_{\text {corr }} / \mathrm{I}_{0}\right) \times 100 \%
\end{aligned}
$$

where $\mathrm{I}_{\text {corr }}$ and $\mathrm{I}_{0}$ were the corrosion current densities of AA5052 covered by polymeric nanofilms and blank, respectively.

From Figure 5 and Table 2, it was seen that the corrosion potentials of blank and electrodeposited polymeric nanofilms modified AA5052 electrodes in the absence and presence of cerium (III) were $-0.620 \mathrm{~V}$, $-0.418 \mathrm{~V}$ and $-0.485 \mathrm{~V}$, respectively. The corrosion current density (Icorr) decreased from $1.21 \times 10^{-3} \mathrm{~A} / \mathrm{cm}^{2}$ for blank electrode to $8.73 \times 10^{-}$ ${ }^{5} \mathrm{~A} / \mathrm{cm}^{2}$ for electrodeposited polymeric nanofilm covered AA5052 without cerium (III). As for cerium-doped polymeric nanofilm covered AA5052 electrode, the Icorr dropped to $5.54 \times 10^{-5} \mathrm{~A} / \mathrm{cm}^{2}$, suggesting that cerium-doped polymeric nanofilm exhibited more favorable inhibition performance. It is assumed that the precipitation of cerium hydroxide or oxide was produced in the vicinity of the cathodic sites, where $\mathrm{OH}^{-}$ions were formed from the oxygen reduction reaction. The protection efficiencies calculated by the expression (2) were determined to be as high as $92.7 \%$ for none cerium-doped polymeric nanofilm, while it was up to $95.5 \%$ for cerium-doped polymeric nanofilm. The result demonstrated that the active corrosion inhibiton effect of cerium incorporated into the polymeric nanofilm exhibited an improvement for the protectiveness of AA5052.

To further investigate the resistance to uniform corrosion of these electrodeposited nanofilms for aluminum alloy surface, the potentiodynamic polarization was conducted in $1.0 \mathrm{M} \mathrm{H}_{2} \mathrm{SO}_{4}$ solution as displayed in Figure 6. The electrochemical parameters of $\mathrm{E}_{\text {corr }}, \mathrm{I}_{\text {corr }}$ and $\beta_{\mathrm{a}}$ and $\beta_{\mathrm{c}}$ are given in Table 3 according to the same method mentioned above. The polarization resistance and protection efficiency were also 


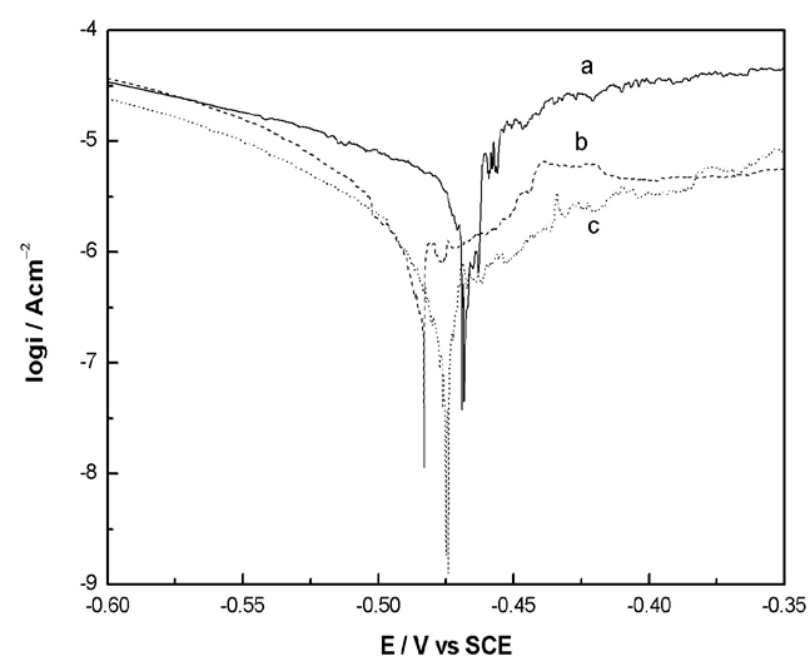

Figure 6: Polarization curves after 20 min exposure in $1.0 \mathrm{M} \mathrm{H}_{2} \mathrm{SO}_{4}$. (a) blank; (b) electrodeposited polymeric nanofilmcovered AA5052 without cerium (III); (c) electrodeposited polymeric nanofilmcovered AA5052 with cerium (III).

\begin{tabular}{|l|l|l|l|l|l|l|}
\hline Sample & $\mathbf{E}_{\text {corr }}(\mathbf{V})$ & $\mathbf{I}_{\text {corr }}\left(\mathbf{A} / \mathbf{c m}^{2}\right)$ & $\boldsymbol{\beta}_{\mathrm{a}}$ & $\boldsymbol{\beta}_{\mathbf{c}}$ & $\mathbf{R}_{\mathbf{p}}\left(\mathbf{\Omega} / \mathbf{c m}^{2}\right)$ & $\mathbf{P E}$ \\
\hline (a) & -0.467 & $1.22 \times 10^{-5}$ & 89.0 & 785.9 & 2934.1 & - \\
\hline (b) & -0.483 & $9.11 \times 10^{-7}$ & 72.5 & 41.8 & 5953.6 & $92.5 \%$ \\
\hline (c) & -0.474 & $6.70 \times 10^{-7}$ & 54.1 & 51.7 & 11023.5 & $94.5 \%$ \\
\hline
\end{tabular}

Table 3: Electrochemical parameters after $20 \mathrm{~min}$ exposure $1.0 \mathrm{M} \mathrm{H}_{2} \mathrm{SO}_{4}$. (a) blank; (b) electrodeposited polymeric nanofilm covered AA5052 without cerium (III); (c) electrodeposited polymeric nanofilm covered AA5052 with cerium (III).

calculated by the above expression (1) and expression (2).

It can be noted that the $\mathrm{E}_{\text {corr }}$ of blank, and electrodeposited polymeric nanofilms modified AA5052 electrodes in the absence and presence of cerium (III) were $-0.467 \mathrm{~V},-0.483 \mathrm{~V}$, and $-0.474 \mathrm{~V}$, respectively. $\mathrm{I}_{\text {corr }}$ of electrodeposited polymeric nanofilm covered AA5052 electrode reduced two orders of magnitude than that of blank electrode. Similarly, $\mathrm{I}_{\text {corr }}$ decreased from $9.11 \times 10^{-7} \mathrm{~A} / \mathrm{cm}^{2}$ for electrodeposited polymeric nanofilm covered AA5052 in the absence of cerium (III) to $6.70 \times 10^{-7} \mathrm{~A} /$ $\mathrm{cm}^{2}$ for cerium-doped polymeric nanofilm covered AA5052 electrode. The protection efficiency of polymeric nanofilm in the absence of cerium (III) for AA5052 was $92.5 \%$, while $94.5 \%$ for cerium-doped polymeric nanofilm coated surface, which was consistent with the result of pitting corrosion. The shift of $\mathrm{E}_{\text {corr }}$ to negative direction and decrease of corr $_{\text {cromonstrated that the corrosion of AA5052 surface was }}$ considerably suppressed by the covering of electrodeposited polymeric nanofilm, and the cerium-doped polymeric nanofilm covered AA5052 exhibited more excellent anticorrosion property than that without cerium. It can be inferred that the coordination between cerium and heterocyclic $\pi$-electron in polymeric nanofilm to prevent corrosive particles to the substrate surface to make corrosion protection of the cerium-doped polymeric nanofilm be improved.

\section{EIS analysis}

In order to estimate the anti-corrosion performance of these polymeric nanofilms, EIS was also employed. Figure 7 presented the bold plot of blank, electrodeposited polymeric nanofilm covered AA5052 electrodes in the absence and presence of cerium (III). It can be seen that the impedance modulus values of polymeric nanofilms covered substrate were higher than that of blank over the frequency range, which demonstrated polymeric coatings could provide corrosion protection for aluminum alloy substrate. Especially in low frequency range, the impedance modulus value of cerium-modified polymeric nanofilm was an order of magnitude higher than that without cerium and two orders of magnitude higher than blank. Generally, phase angle was rather related to the nanofilm characteristics and anti-corrosion property of substrate and the higher phase angle implied good uniform corrosion resistance. For cerium-doped polymeric nanofilm, the phase angle was always approaching to $-90^{\circ}$ in a wide range of frequency and its phase shifts reduced less than that of blank and electrodeposited polymeric nanofilm in the absence of cerium from medium to low frequency, which also indicated that the stability of the cerium-doped nanofilm was improved.

Meanwhile, the impedance could be evaluated by the radius of the capacitive loops in Nyquist plots as shown in Figure 8. It was found that no matter whether cerium or not, polymeric nanofilms covered substrate surface had significantly higher impedance than blank.

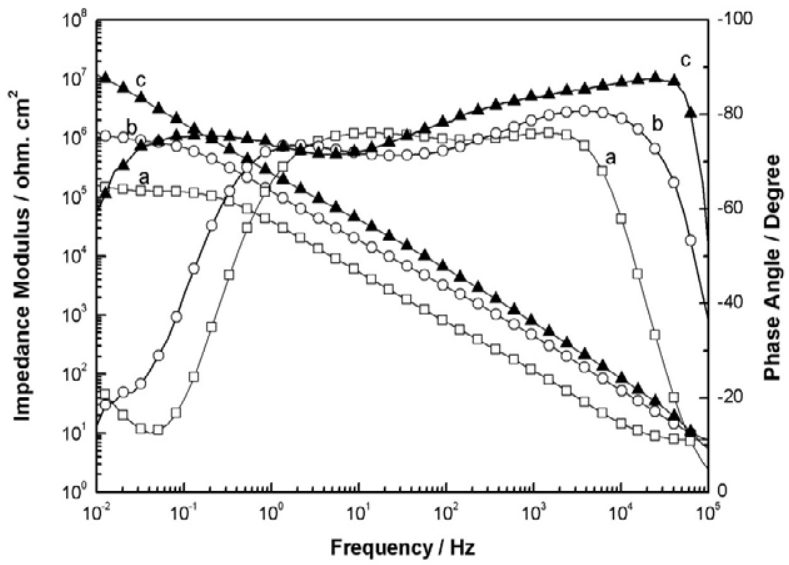

Figure 7: Bode plots of AA5052 surface after 1 hour exposure in $0.1 \mathrm{MNaC}$ solution. (a) blank; (b) electrodeposited polymeric nanofilmcovered AA5052 without cerium (III); (c) electrodeposited polymeric nanofilmcovered AA5052 with cerium (III)

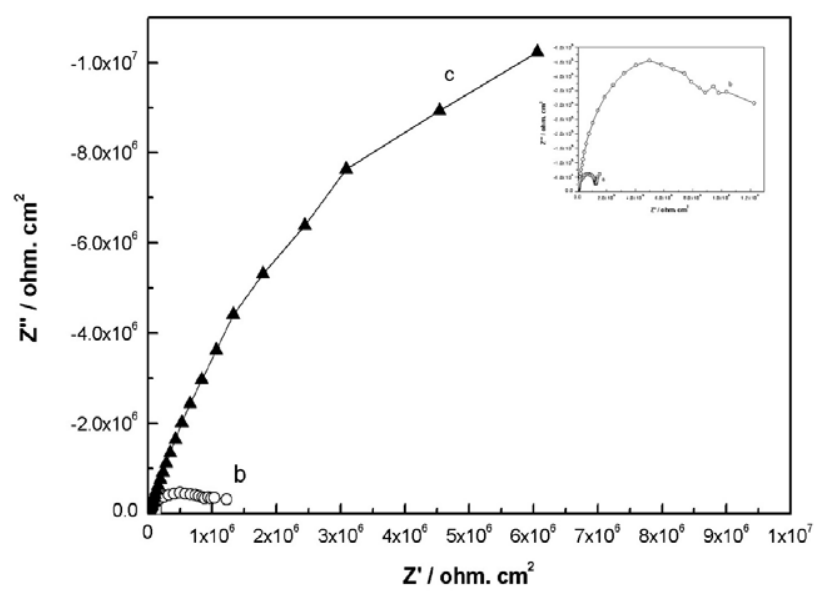

Figure 8: Nyquist plots of AA5052 surface after 24 hours exposure in 0.1 $\mathrm{MNaCl}$ solution. (a) blank; (b) electrodeposited polymeric nanofilmcovered AA5052 without cerium; (c) electrodeposited polymeric nanofilmcovered AA5052 with cerium. 


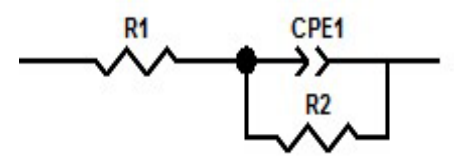

Figure 9: Equivalent circuit used for numerical fitting of the impedance spectra.

\begin{tabular}{|l|l|l|l|l|}
\hline Sample & $\mathbf{R}_{\mathbf{1}}\left(\mathbf{\Omega} \cdot \mathbf{c m}^{2}\right)$ & $\mathbf{C P E} 1-\mathbf{T}\left(\mathbf{F} / \mathbf{c m}^{-2} \cdot \mathbf{s}^{-\mathbf{n}}\right)$ & $\mathbf{C P E} 1-\mathbf{P}$ & $\left.\mathbf{R}_{\mathbf{2}} \mathbf{(} \mathbf{\Omega} \cdot \mathbf{c m}^{\mathbf{2}}\right)$ \\
\hline (a) & 6.403 & $4.75 \times 10^{-6}$ & 0.87387 & $1.405 \times 10^{5}$ \\
\hline (b) & 1.883 & $1.57 \times 10^{-6}$ & 0.84512 & $1.173 \times 10^{6}$ \\
\hline (c) & -0.989 & $7.74 \times 10^{-7}$ & 0.86545 & $3.392 \times 10^{7}$ \\
\hline
\end{tabular}

Table 4: EIS parameters by fitting the experimental data. (a) blank; (b) electrodeposited polymeric nanofilm covered AA5052 without cerium (III); (c) electrodeposited polymeric nanofilm covered AA5052 with cerium (III).

Moreover, compared with electrodeposited polymeric nanofilm in the absence of cerium, the cerium-modified nanofilm presented larger capacitive loops, which meant that cerium-modified nanofilm had more effective anti-corrosion property. The EIS result was consistent with the $\mathrm{R}_{\mathrm{p}}$ value of potentiodynamic polarization.

In order to explain the mechanism of corrosion protection, EIS results could be numerical fitted by equivalent circuit. Figure 9 displayed the model of EIS spectra. $\mathrm{R}_{1}$ was the simulation of the solution resistance, the constant phase element (CPE1) was responsible for the film capacitance and $\mathrm{R}_{2}$ accounted for polarization resistance, respectively. The values of $R_{1}, R_{2}$ and total capacitance $(T)$ of the CPE1 were calculated by fitting the experimental data using ZView software as listed in Table 4 . The $R_{2}$ value was significantly relied on the property of these polymeric nanofilms and intensely dependent on anti-corrosion performance of the nanofilms. So the higher $R_{2}$ value represented good uniform anti-corrosion performance. It was clearly observed that the $R_{2}$ value of cerium-modified polymeric nanofilm was the highest, implying that the film was the most compact and the corrosion resistance of electrodeposited polymeric nanofilm of DBN could be improved by doping a certain amount of cerium. On the basis of the above results, it could be assumed that cerium as corrosion inhibitor did enhance anti-corrosion performance of electrodeposited polymeric nanofilm of DBN.

\section{Conclusion}

The cerium-modified polymeric nanofilm of DBN was successfully prepared by two-step potential electrodeposition onto AA5052 substrate surface. WCA of aluminum alloy surface covered by ceriummodified polymeric nanofilm was higher, which exhibited a certain the hydrophobicity. The electrochemical measurements of OCP, potentiodynamic polarization and EIS demonstrated that the substrate surface covered by cerium-doped polymeric nanofilm had remarkable anti-corrosion property. Cerium as corrosion inhibitor does improve the corrosion resistance of polymeric nanofilm and further studies are necessary to fully understand the mechanism of $\mathrm{Ce}\left(\mathrm{NO}_{3}\right)_{3}$.

\section{Acknowledgement}

This work was supported by the Fundamental Research Funds for the Central Universities (No. QN2013085) and the National Natural Science Foundation of China (No. 21203152).

\section{References}

1. Mori K, Sasaki H, Kobayashi I, Sai S, Hirahara H, et al. (2000) Adhesion of nylon-6 to triazine trithiol-treated metals during injection molding. J Adhes Sci Technol 14: 791-803.
2. Mori K, Asakura T, Oishi Y (2003) Electrochemical Reaction in the Polymer Plating of Triazine Dithiols. Polym J 35: 568-572.

3. Kang Z, Li Y, Mori K (2005) Application of polymer plating to inhibit corrosion of magnesium alloy. Mater Sci Forum 488: 661-664.

4. Wang F, Mori K, Oishi Y (2006) Electrochemical Polymerization of 6-(N-Allyl1,1,2,2-tetrahydroperfluorodecyl)amino-1,3,5-triazine-2,4-dithiol Monosodium on Aluminum. Polym J 38: 484-489.

5. Silikas N, Wincott P, Vaughan D, Watts D, Eliades G (2006) Surface characterization of precious alloys treated with thione metal primers. Dent Mater 23: 665-673

6. Wang F, Luo H, Wang Q, Wang J, Xu J (2009) Preparation of Superhydrophobic Polymeric Film on Aluminum Plates by Electrochemical Polymerization. Molecules 14: 4737-4746.

7. Bof Bufon CC, Heinzel T, Espindola P, Heinze J (2010) Influence of the Polymerization Potential on the Transport Properties of Polypyrrole Films. J Phys Chem B 114: 714-718.

8. Wang F, Wang Y, Li Y, Wang Q (2011) Preparation of triazinedithiol polymeric nanofilm by two-step potentiostatic polymerization technique on aluminum surface. Materials Lett 65: 621-623.

9. LiY, Wang D, Zhang H, Wang F (2011) Study on Triazinethiol Electropolymerized Films Prepared by Cyclic Voltammetry and Galvanostatic on Copper Alloy Surface. Int J Electrochem Sci 6: 4404-4410.

10. Wang F, Liu J, Li Y, Fan R (2012) Complex Barrier Layer of Triazinedithoil Prepared by Electrodeposition and Initiated Polymerization on Aluminum Alloy towards Corrosion ProtectionInt. J Electrochem Sci 7: 3672-3680.

11. Wang F, Liu J, Li Y, Wang Y (2012) Novel composite nanofilm of electropolymerization and self-assembling on AA5052 surface as anticorrosion coating. J Appl Polym Sci 123: 2906-2910.

12. Baba H, Kodama T (1999) Corrosion Inhibition and Characteristics of the Triazinedithiol Surface Film on Copper Under Potentiostatic Anodization. Corros Sci 41: 1987-2000.

13. Mori K, Sasaki Y, Sai S, Kaneda S, Hirahara H, et al. (1995) Electrochemical Polymerization of 2-(Dioctylamino)-1,3,5-Triazine-4,6-Dithiol on Iron Plates. Langmuir 11: 1431-1434.

14. Wu L, Liu L, Li J, Hu J (2010) Electrodeposition of cerium (III) -modified bis[triethoxysilypropyl] tetra-sulphide films on AA2024-T3 (aluminumalloy) for corrosion protection. Surf Coat Tech 204: 3920-3926.

15. Dias SAS, Lamaka SV, Nogueira CA, Diamantino TC, Ferreira MGS (2012) Sol-gel coatings modified with zeolite fillers for active corrosion protection of AA2024. Corros Sci 62: 153-162.

16. Fahrenholtz WG, O'Keefe MJ, Zhou HF, Grant JT (2002) Characterization of cerium-based conversion coatings for corrosion protection of aluminum alloys. Surf Coat Tech 155: 208-213.

17. Cambon JB, Esteban J, Ansart F (2012) Effect of cerium on structure modifications of a hybrid sol-gel coating, its mechanical properties and anticorrosion behavior. Mat Res Bull 47: 3170-3176.

18. Cere S, Pepe A, Aparicio M, Duran A (2006) Cerium hybrid silica coatings on stainless steel AISI 304 substrate. J Sol-Gel Sci Technol 39: 131-138.

19. Wang $\mathrm{H}$, Akid $\mathrm{R}$ (2008) Encapsulated cerium nitrate inhibitors to provide highperformance anti-corrosion sol-gel coatings on mild Steel. Corros Sci 50: 1142-1148.

20. Wang F, Mori K, Kang ZX, Oishi $Y(2007)$ Magnetic field effects on the polymerization of 6-N,N-dioctylamino-1,3,5-triazine-2,4-dithiol. Heteroatom Chem 18: 60-64.

21. Hou W, Kang Z (2013) Preparation of Duplex Film through Microarc Oxidation Coloring and Polymer Plating on Mg-Li Alloy and Its Corrosion Resistance. Int J Electrochem Sci 8: 5613-5620.

22. El-Etre AY (1998) Natural honey as corrosion inhibitor for metals and alloys. i. copper in neutral aqueous solution. Corros Sci 40: 1845-1850. 